\title{
Exploring Strategies and Guidelines for Developing Full Body Video Game Interfaces
}

\author{
Juliet Norton \\ School of EECS \\ University of Central Florida \\ 4000 Central Florida Blvd. \\ Orlando, FL 32816 \\ juliet@knights.ucf.edu
}

\author{
Chadwick A. Wingrave \\ School of EECS \\ University of Central Florida \\ 4000 Central Florida Blvd. \\ Orlando, FL 32816 \\ cwingrav@cs.ucf.edu
}

\author{
Joseph J. LaViola Jr. \\ School of EECS \\ University of Central Florida \\ 4000 Central Florida Blvd. \\ Orlando, FL 32816 \\ jjl@eecs.ucf.edu
}

\begin{abstract}
We present a Wizard-of-Oz study exploring full body video game interaction. Using the commercial video game Mirror's Edge, players are presented with several different tasks such as running, jumping, and climbing. Following our protocol, participants were given complete freedom in choosing the motions and gestures to compete these tasks. Our experiment results show a mix of natural and constrained gestures adapted to space and field of view restrictions. We present guidelines for future full body interfaces.
\end{abstract}

\section{Categories and Subject Descriptors}

H.5.2 [Information Interfaces and Presentation]: User Interfaces - interaction styles; K.8 [Personal Computing]: Games

\section{General Terms}

Full Body Interfaces, Video Games

\section{Keywords}

\section{INTRODUCTION}

Spatially convenient input devices [21], such as the Nintendo Wii Remote (Wiimote) and Microsoft's upcoming Project Natal, enable the design and creation of full-body interfaces for video games. These devices collect information about a player's real-world movement to enable game designers to implement 3D techniques for interaction tasks such as travel, selection and manipulation [5]. While these devices can potentially provide more engaging experiences, they also give players more freedom when interacting with a video game, providing a more natural, healthy and immersive gameplay experience. However, a challenge with full body game interfaces is that there are not always mappings from user action to game response for the following reasons:

- Video games present new experiences, where players do not always know which action to take such as skating a half-pipe or snow boarding.

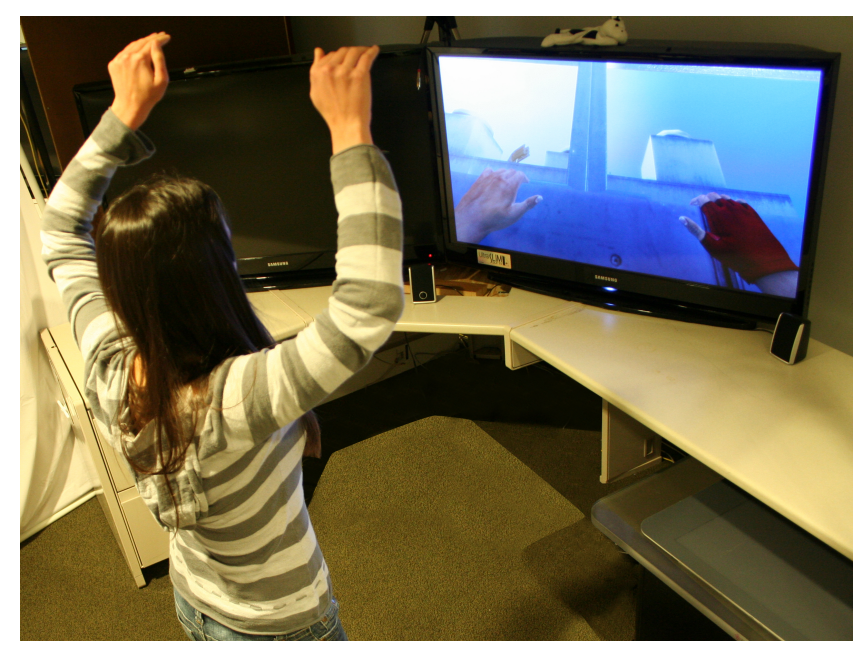

Figure 1: Players are able to explore and experiment video game control without constraints for full body interaction. Here, a player performs a jump/high gesture to climb onto a platform.

- Video game actions do not always have real-world counterparts such as casting spells or working alien technology.

- User actions are limited by space and physics; walking runs out of room, game walls are not tangible and ladders have no real-world rungs for the player to step.

- Video game controllers constrain the player but full body interfaces have no such constraints, and it is unclear which real world affordances are most important to the player.

- Not all full body interaction is fun and accounts need to be made for issues such as exhaustion, injury avoidance and boredom.

Therefore, to meet this challenge, important questions such as what full body actions will players use, what full body actions are usable, and how can we build natural and compelling full body video game interfaces, must be addressed.

We explored these questions using the commercial video game Mirror's Edge, a first person action-adventure video game incorporating Parkour-like methods of travel as well as basic fighting motions. This requires the player to run, jump, climb, and duck on building rooftops. Similar to past 
user centered design studies of 3D UI's [22], we look to see how users adapt to difficult situations and analyze the strategies they form. To develop a natural and unbiased full body interface, players were given complete freedom in choosing appropriate symbolic control motions. This Wizard-of-Oz method [7] uses the experiment moderator as the participant's interpreter and controller of the video game. In this way, the focus is on the player's intent and the methodology allows them to explore different gestures to express this intent. By analyzing the video recordings and data collected by post-experiment semi-structured interviews, we collected themes and generated guidelines regarding full body video game interfaces.

In the next section, we discuss work related to full body interaction and gestural user interfaces (UI). Section 3 provides a brief description of Mirror's Edge and why it was chosen for our study. Section 4 describes our experimental design and methodology. Section 5 presents results, discusses our findings and proposes a full body interface for Mirror's Edge, Finally, Section 6 concludes the paper.

\section{RELATED WORK}

3D UIs have been extensively researched in the realm of Virtual Reality and the search for basic interaction and travel techniques has been well explored $[2,5]$. Prior work in 3D UIs for gaming has been dominated by hand-arm gestures [14] and mimicking real world actions [1]. Commercial games for the Nintendo Wii have been released that use gestures in order to play sporting games such as tennis. Unfortunately due to noise in accelerometer based tracking and variance in natural motion, most of these games can be played and sometimes work better when an unnatural wrist flick is used instead of the real world human action. The game Wizards uses Hidden Markov Models to reduce this noise and variance, but the gestures are only arm-based and are used to cast spells which is not a normal human activity [12].

Some full body control applications have been developed in the VR and game industries. The ALIVE system allows for unencumbered full body motion for interaction with an autonomous agent, but the interaction is slow and simple and the body is only used for context of only a few simple arm gesture [13]. Alternatively, PingPongPlus is a very physical Augmented Reality application allowing the user to exert natural effort and performance techniques, but only the ball required trackingc̃ite303115. More recently full body games and travel techniques have been developed that utilize the Nintendo Wii Remote for inexpensive accelerometer-based tracking of the limbs $[8,16]$.

There exists little research on travel using full body control for video game applications [16]. Commercial games such as Sean White's Snowboarding for the Nintendo Wii allows use of the body for steering in the game via the Wii Fit. This navigation is restricted in freedom of direction and speed as there is a general down hill motion that naturally occurs. Also, the game still requires the Wii Remote for interactions such as tricks. Although 3D travel techniques in gaming research is sparse, extensive research in travel has been done in the field of Virtual Reality [5], including physical travel techniques such as Seven League Boots [10], locomotion devices[11], and affects on presence[17]. Steering is an essential element of travel for which there are many established techniques ranging from most precise to fastest to use $[3,4]$. RealNav explores 3D locomotion using gaming hardware [20].

\section{MIRROR'S EDGE}

Mirror's Edge is a commercial action-adventure game published by Electronic Arts in 2007. The player controls a character named Faith that completes objectives by moving through the environment in a Parkour type fashion. Parkour is a non-competitive sport originating in urban France that requires the athlete to traverse an environment and its obstacles using only the human body [9]. In this game, the environment is primarily the rooftops of an urban setting with obstacles including fences, railings, walls, and pipes. The game requires creativity, balance, precision and speed. To complete the illusion of control over Faith, Faith's arms and legs are shown at times of interactions with the environment to convey the Parkour feel and motion. The tasks involved include: running, jumping, climbing, balance walking, wall running, sliding, combat, and opening doors.

\section{EXPERIMENT}

This study is an initial look into designing a travel intensive full body motion video game. It is therefore an exploratory study purposed to form an understanding of what is required for an engaging full body motion video game. This foundational information will then allow development of interaction techniques tested in future usability experiments.

\subsection{Experimental Task}

The participants played through the first level of Mirror's Edge using full body interaction rather than hand operated controls. The objective of this level was to deliver a package to an NPC teammate at another rooftop location in the city. Their path to the objective was linear, but vast, with a variety of ways to traverse the terrain. Actions the participants can or may be required to perform are: running, jumping, sliding, balancing, climbing, combat, and opening doors. The pace at the beginning of the level is at the participant's leisure, but mid-way through, a chase begins and the participant must move faster to avoid being killed by NPC shooters.

\subsection{Experimental Design and Procedure}

Upon entering the lab, participants reviewed an informed consent form describing the experiment. Next, a gaming and Parkour experience pre-questionnaire was filled out and then participants were then shown a video ${ }^{1}$ of the first level of Mirror's Edge. This was followed by the experiment moderator explaining basic game mechanics, including specific interactive elements, directions, and employable actions. Once the participants understood their objective, they warmed up with the experiment moderator by performing jumping jacks. This was used to reduce social inhibition as well as warm-up the participant for the interaction. The experiment moderator then left the participant in the space, and sat at the control desk, telling the participant to begin.

\footnotetext{
${ }^{1}$ Mirror's Edge Walkthrough - Prologue: Financial District http: //www . youtube. com/watch?v=UpbU4o1E480
} 


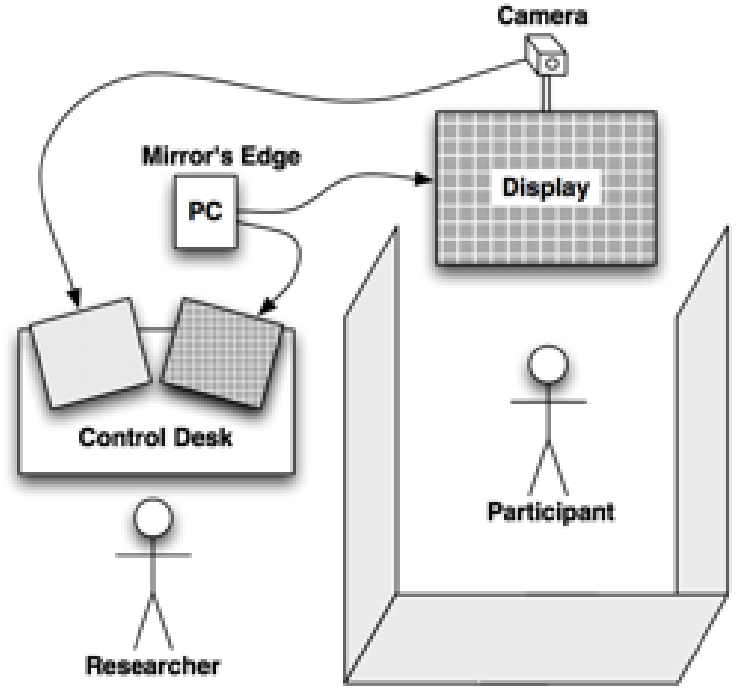

Figure 2: The participant played in the enclosed space. A video feed of both the game and the participant was sent to the control station where the experiment moderator played the game.

Participants were given complete freedom in choosing appropriate motions to play the first level of Mirror's Edge. This activity was performed until they completed the level or up to fifteen minutes. The participant was not tracked by any tracking system. Instead, the participant's motions were translated into the game by a Wizard-of-Oz approach, with the experiment moderator playing the game based upon the user's actions such as gestural motions, facial expression, and vocalization. For example, if the participant stepped back for more space in the physical world, Faith would also be commanded to take a step back. After completing the game session, the participants were given a post-experiment semi-structured interview using a questionnaire that gauged their experience using full body motion, their level of presence in the scenario, and preference over a controller if applicable. The semi-structured interview approach was used to elicit more discussion than a conventional questionnaire.

\subsection{Participants and Apparatus}

Fourteen participants (five female, nine male) from a variety of backgrounds including computer science and digital arts majors, aged 21 to 30, participated in the exploratory experiment. Participants were recruited by word of mouth from the University of Central Florida. From the pre-questionnaire, five had played the game or demo, and twelve had played a full body game in the past such as Dance Dance Revolution. There were, however, only two that had used full body movement for navigation in games such as Top Skater. Nine participants preferred gesture control rather than a handoperated input device for natural three-dimensional tasks such as sports, dancing, and shooting. Ten participants had experience in sports that require great amounts of coordination and five did not know what Parkour was, but twelve had experienced Parkour type activities. The experimental duration lasted no longer than one hour, and each participant was compensated 10 dollars.
The experimental setup consisted of a desktop PC with a 2.66 GHz Intel Core i7-920 processor and a Nvidia 260 GTX graphics card running Windows 7 . The visual display was a 50 inch 1920x1080 resolution Samsung DLP 3D HDTV display with a refresh rate of $60 \mathrm{~Hz}$. Stereo desktop speakers were used to output sound from the computer. The experiment space (see Figure 2) was approximately 10' by 10' and enclosed by a curtain for privacy and distraction reduction, both important for reducing the potential impact to the study of social inhibition [23]. The "wizard's" control desk was stationed outside of the curtain, again to reduce social inhibition. This desk had a duplicate view of the game as well as a video feed of the participant from a video camera perched above the participant's display. This camera also recorded the experiment and post-experiment discussion for later analysis. One issue with the video feed was that the researcher had to flip the right from left and this was trained for in multiple piloted experiments.

\subsection{Social Inhibition Reduction}

As this experiment seeks unconstrained participant motions for full body video game control, a detrimental effect is social inhibition [23]. Social inhibition in this experiment potentially retards the participant from full movement and engagement in the video game. The causes of this inhibition include being in an unfamiliar setting, interacting with an unfamiliar experiment moderator, being in a "sterile" laboratory setting, being watched and being video taped. These potentially can impact the participant's behavior and curb how they play the game. To account for this, several procedures were used to reduce this effect. These include a participant recruitment process that focused on word of mouth over general advertisements, the use of a walled-off experimental space that removes even the experiment moderator from view, and pre-experiment exercise to break the social norms of movement in public spaces.

\subsection{Methodology}

Using the Wizard-of-Oz methodology, the experiment moderator operated as the interpreter of participant behavior, translating their motions into Mirror's Edge actions. Because of this, the participants were free to explore multiple methods of control, so long as their actions were understandable at least by the experiment moderator. In addition, after the game, the experiment moderator asked non-leading questions about their behavior (e.g. questions that ask what sort of gestures were performed, how and why they performed them and how they contrasted to other techniques they tried [18]. This enabled the participant an opportunity to rationalize their behavior and give further insight to the experiment moderator. By recording these sessions, an analysis and coding of the video was performed, first by taxonomic and analysis, noting body positions and motions when participants attempted each Mirror's Edge task. This process was then iterated, modifying the taxonomy until it represented the observed participant behavior and then thematic analysis was performed to reduce the dimensionality.

\section{RESULTS}

We present our findings in terms of the video and interview. Following this, we discuss the identified themes, guidelines and resulting gesture-based interface. 


\begin{tabular}{|c|c|c|}
\hline \multicolumn{3}{|c|}{ Post-Questionnaire } \\
\hline & Question & Mean \\
\hline Q1 & $\begin{array}{l}\text { Using full body motion to play the level was } \\
\text { tiring. }\end{array}$ & $\begin{array}{l}2.50 \\
(1.22 \sigma)\end{array}$ \\
\hline Q1 & $\begin{array}{l}\text { I wouldn't play more than a level at a time } \\
\text { using full body motion because it would be } \\
\text { too tiring. }\end{array}$ & $\begin{array}{l}4.14 \\
(1.17 \sigma)\end{array}$ \\
\hline Q3 & $\begin{array}{l}\text { Freedom to create my own gestures made it } \\
\text { easy to play the game because I didn't have } \\
\text { to learn or remember pre-defined gestures or } \\
\text { controls. }\end{array}$ & $\begin{array}{l}1.93 \\
(0.73 \sigma)\end{array}$ \\
\hline Q4 & $\begin{array}{l}\text { I would have preferred performing specified } \\
\text { gestures rather than coming up with my own. }\end{array}$ & $\begin{array}{l}3.70 \\
(1.38 \sigma)\end{array}$ \\
\hline Q5 & $\begin{array}{l}\text { I felt pressured when coming up with gestures } \\
\text { on the spot. }\end{array}$ & $\begin{array}{l}3.57 \\
(1.34 \sigma)\end{array}$ \\
\hline Q6 & $\begin{array}{l}\text { I was easily able to come up with gestures to } \\
\text { perform the task at hand in the game. }\end{array}$ & $\begin{array}{l}1.71 \\
(0.91 \sigma)\end{array}$ \\
\hline Q7 & $\begin{array}{l}\text { I felt confused and uncoordinated when per- } \\
\text { forming the gestures. }\end{array}$ & $\begin{array}{l}3.92 \\
(1.32 \sigma)\end{array}$ \\
\hline Q8 & $\begin{array}{l}\text { I would play the entire game using full body } \\
\text { motion because I feel more connected to the } \\
\text { action and story. }\end{array}$ & $\begin{array}{l}1.86 \\
(1.10 \sigma)\end{array}$ \\
\hline Q9 & $\begin{array}{l}\text { Using full body motion was exciting because } \\
\text { I felt like I accomplished Faith's tasks. }\end{array}$ & $\begin{array}{l}1.21 \\
(1.57 \sigma)\end{array}$ \\
\hline Q10 & $\begin{array}{l}\text { I was afraid to jump at the ledge of a building } \\
\text { because I was using my body instead of a } \\
\text { controller. }\end{array}$ & $\begin{array}{l}4.29 \\
(1.20 \sigma)\end{array}$ \\
\hline Q11 & $\begin{array}{l}\text { I felt as if I was in the game because the } \\
\text { avatar was performing the same actions as } \\
\text { my body. }\end{array}$ & $\begin{array}{l}1.86 \\
(1.17 \sigma)\end{array}$ \\
\hline Q12 & $\begin{array}{l}\text { If the game responded perfectly to my move- } \\
\text { ment, I'd use the system. }\end{array}$ & $\begin{array}{l}1.00 \\
(0.00 \sigma)\end{array}$ \\
\hline Q13 & $\begin{array}{l}\text { If the game responded perfectly to my move- } \\
\text { ment, I'd prefer to play the game with full } \\
\text { body motion. }\end{array}$ & $\begin{array}{l}1.36 \\
(0.84 \sigma)\end{array}$ \\
\hline \multicolumn{3}{|c|}{ Additional Experienced Player Questions } \\
\hline Q14 & $\begin{array}{l}\text { I didn't feel any more present in the game } \\
\text { than if I had been using a controller. }\end{array}$ & $\begin{array}{l}4.60 \\
(0.89 \sigma)\end{array}$ \\
\hline Q15 & $\begin{array}{l}\text { Using my body to play the game was harder } \\
\text { work in terms of control than using a con- } \\
\text { troller. }\end{array}$ & $\begin{array}{l}2.40 \\
(1.95 \sigma)\end{array}$ \\
\hline Q16 & $\begin{array}{l}\text { Using my body to move through the VE took } \\
\text { less concentration than using a controller. }\end{array}$ & $\begin{array}{l}3.20 \\
(1.79 \sigma)\end{array}$ \\
\hline Q17 & $\begin{array}{l}\text { Learning to use my body to move through the } \\
\text { VE is easer than learning to use a controller. }\end{array}$ & $\begin{array}{l}1.20 \\
(0.44 \sigma)\end{array}$ \\
\hline
\end{tabular}

Table 1: Post-Questionnaire Mean and Standard Deviation. Scoring on Likert scale ( $1=$ Strongly Agree; 5 = Strongly Disagree)

\subsection{Video}

The observed participant-controlled motions were organized into three general tasks: travel, gaze control, and travel gestures. Travel is further divided into locomotion and steering subtasks. Locomotion is the subtask where a player moves their virtual representation through the virtual space. This is further split into natural locomotion, where realistic user motions map to Faith's locomotion, and compensating locomotion, where physically constrained participant motions map to Faith's locomotion. The steering subtask involves a change in the direction of locomotion. This is differentiated from gaze control, which is the task of changing Faith's viewpoint to look around the environment. Lastly, travel gestures are pantomimic gestures mapped directly to spe-
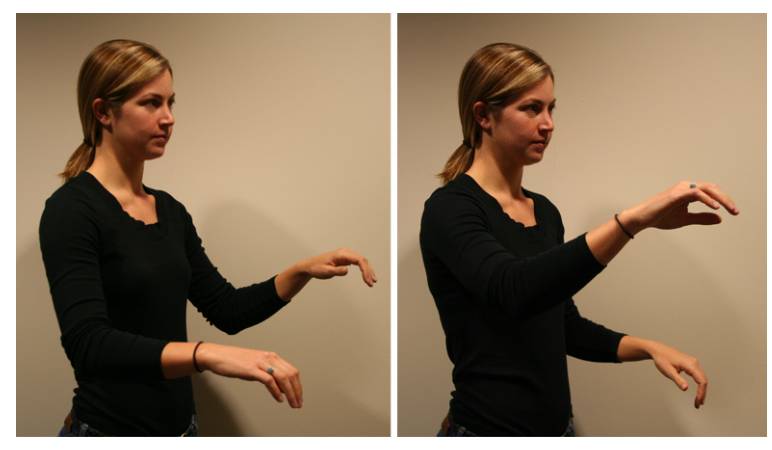

Figure 3: The participant made a doggie paddle type movement to symbolize locomotion.

cialized travel actions, with the exception of sliding and wall running which are symbolic gestures due to their complex nature.

In this travel intensive video game, locomotion is a dominant task and is highly constrained by the limited physical space of the real world. For this reason, compensating locomotion plays a larger role than the natural locomotion, which can only be used sparingly. Natural locomotion motions include physically walking forwards or backwards in relation to the display or strafing by walking side to side. In the experiment, nine participants initially used natural locomotion gestures (see Table 2). Seven of these quickly switched to compensating locomotion, such as running in place, with the remaining two discovering compensating locomotion after the chase had begun. Until then, they used natural locomotion to walk to the display and then walked backwards to start walking forward again. This was ambiguous to the experiment moderator because the participant could also mean walk backwards, the participants were frustrated with this approach. Conversely, running in place, a compensating mimetic gesture, was used by all participants at some point (Table 2). Some participants experimented with multiple gestures, to identify what best suited them. Two other compensating gestures observed were arm based (they did not involve legs): a doggie paddle arm metaphor (see Figure 3), and the natural arm-swing during walking or running.

Unlike locomotion, steering gestures fit into the physical environment but suffered from field of regard issues stemming from the display's fixed location. Three participants utilized a natural steering gesture (i.eturning forward direction point away from the display in accordance to Faith's orientation) throughout the experiment and often found themselves unable to see the display (see Table 2). Three others experimented with gestures and settled on compensating gestures after their backs were turned to the screen. Shoulder twist $(57 \%)$ and body rotate and return $(79 \%)$ were the most common, with seven participants using them interchangeably. Torso lean to side was attempted (29\%), but was replace by a shoulder twist or body rotate and return to center gesture. Half of the participants that used their arms for directional movement in the gaze control task also used these gesture to steer. One individual who used arm swing translation coupled this with a hip and arm swing in the direction of desired turning. Two participants used an unusual but ef- 


\begin{tabular}{|l|l|c|}
\hline \multicolumn{3}{|c|}{ Travel Gestures } \\
\hline Task & Techniques & $\%$ of Total \\
\hline Natural & displacement step (extended) & $28 \%$ \\
Locomotion & displacement step (local) & $100 \%$ \\
\hline Natural & rotate to new center & $42 \%$ \\
Steering & & \\
\hline \multirow{3}{*}{ Compensating } & tread in place (extended) & $100 \%$ \\
Locomotion & tread in place (local) & $21 \%$ \\
& doggie paddle & $7 \%$ \\
& arm swing & $14 \%$ \\
\hline \multirow{3}{*}{ Compensating } & shoulder twist & $57 \%$ \\
& hip-arm swing & $7 \%$ \\
& one arm frog swim & $14 \%$ \\
& body rotate and return & $79 \%$ \\
& torso lean to side & $29 \%$ \\
\hline
\end{tabular}

Table 2: The observed travel gestures were organized into a taxonomy of natural/compensating strategies and locomotion/steering subtasks. Using these gestures, participants were able to express their intent to the experiment moderator.
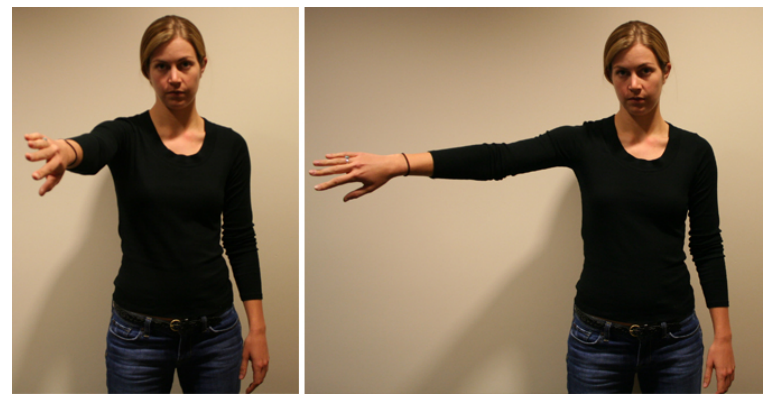

Figure 4: The participant's arm is moved in a frogswim like movement to indicate turning in the corresponding direction.

fective frog-swim like metaphor for steering (see Figure 4).

Participants controlled their viewpoint differently depending on if they intended to look around the video game world (gaze control) or if they were locomoting (orientation). Gaze control was the first action participants attempted once the experiment began. Twelve participants initially attempted this by turning their head. While head turning is a natural and fast, it has a problem that players found themselves looking away from the display. To compensate, eleven of the twelve head turners eventually paired this technique with the body rotate and return to center compensation steering technique. Some also experimented with head turning accompanied with pointing or verbal instructions (see Table 3). Directional arm movement was an imprecise pointing type movement that was not paired with head turning.

Participants performed pantomimic gestures (i.e. a one-toone correspondence between the gesture and the video game action) [19] for: jumping, climbing, combat, balancing, and door opening (see Table 4). Medium jumping, sliding, and wall running were represented with symbolic gestures (i.e. gestures that are represent an interaction that cannot be performed as in reality because of interface limitations) due

\begin{tabular}{|l|c|}
\hline \multicolumn{2}{|c|}{ Gaze Control Gestures } \\
\hline head turn & $86 \%$ \\
\hline point & $14 \%$ \\
\hline verbal & $29 \%$ \\
\hline directional arm movement & $29 \%$ \\
\hline
\end{tabular}

Table 3: Gaze control allowed the participants to look around the video game and was distinct from the steering task. Most participants tried head turning but settled on body rotate and return to center, a technique also commonly used for steering.

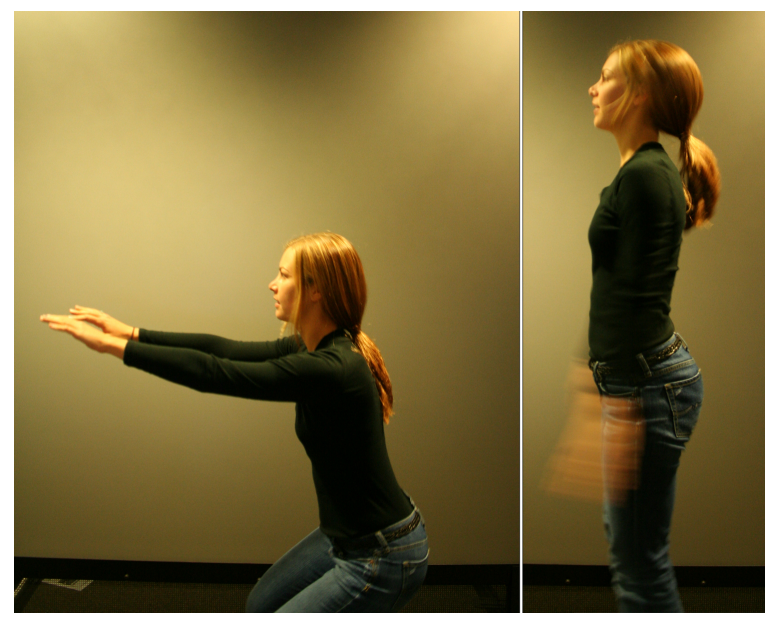

Figure 5: For medium jumps the participants stuck their arms out in front then pushed down to symbolize vaulting over the objects.

to their need for space, props, and athleticism. Jumping was split into three jumping tasks: low, medium and high. During low jumping, jumping to another platform of equal or lower height, 13 participants executed a normal hop without using their arms. The fourteenth performed these jumps as she did medium jumps (see Figure 5), where medium jumps are jumps over obstacles lower than head height, such as fences and air conditioning units. Eleven participants performed this gesture. High jumps, jumps with arms straight up, were performed to reach up and climb on top of obstacles. These were performed with hops and with arms straight-up over-head by ten of the participants.

Climbing was split into three climbing tasks: pole, from hanging and ladder. Eleven participants executed climbing a pole by putting one arm over the other. The remaining participants either jumped or executed a forward locomotion gesture. Climbing up from a hanging state, or up over a fence, was executed by an "arms push down" gesture, where participants held their arms up, then pushed them down to their hips. This was performed by nine participants (see Figure 1). Seven participants performed the ladder climbing task using alternating arms, similar to a pole climb.

For the remaining tasks, participants performed various gestures and movements. All participants who entered combat performed punch gestures, but two participants also kicked. All participants leaned their bodies back and forth to signify balance when walking on a beam, but ten participants also held their arms in a T-shape. Most participants did not at- 


\begin{tabular}{|c|c|c|}
\hline \multicolumn{3}{|c|}{ Travel Gestures } \\
\hline Action & Techniques & $\%$ of Total \\
\hline \multirow{3}{*}{ Jump } & low: hop & $93 \%$ \\
\hline & medium: hop, arms out & $79 \%$ \\
\hline & hight: hop, arms up & $71 \%$ \\
\hline \multirow{3}{*}{ Climb } & pole: arm over other & $78 \%$ \\
\hline & from hang: arms push down & $64 \%$ \\
\hline & ladder*: alternating arms & $50 \%$ \\
\hline \multirow{2}{*}{ Combat* } & punch & $78 \%$ \\
\hline & kick & $14 \%$ \\
\hline Slide* & duck & $21 \%$ \\
\hline \multirow{2}{*}{ Balance } & arms out, lean & $71 \%$ \\
\hline & no arms, lean & $29 \%$ \\
\hline \multirow{2}{*}{ Wall Run* } & side arm paddle & $7 \%$ \\
\hline & side jump & $21 \%$ \\
\hline \multirow{5}{*}{ Door Open } & turn handle & $50 \%$ \\
\hline & kick & $50 \%$ \\
\hline & punch & $21 \%$ \\
\hline & pound & $7 \%$ \\
\hline & push & $14 \%$ \\
\hline
\end{tabular}

Table 4: Participant gestures for common interactions in environment. *Note that for climbing ladders, sliding, wall running, and combat not all participants engaged in these activities.

tempt a wall run but the ones that did used a doggie paddle arm gesture, and the rest jumped with their feet angled in the direction of contact with the wall. The two most common door opening gestures were handle turning and door kicking (seven participants each). Punching, pounding, and pushing the door gestures were also used. Interestingly, six participants switched to a kick gesture for opening the door. We believe this is because the participants adjusted their gesture set to what the saw Faith do on screen as would be expected due to visual dominance [6].

\subsection{Post-Experiment Interview}

The following themes were identified in the interview.

Individual Fatigue. On average participants agreed that playing the level with full body motion was tiring, but not so tiring that they wouldn't play more than a level.

Engagement. Most participants felt connection to the action occurring in the game. The felt the full body motion made the game exciting and felt as if they were in the game. One participant was afraid to take leaps between rooftops and others were hesitant regarding combat with another human.

Gesture Creation. On average participants liked the freedom of choice to create their own gestures, didn't feel significant pressure to do so, and were not privy to the idea of preset gestures. They felt it was easy to come up with these gestures and did not feel confused or uncoordinated when performing them. All participants said they would use the system if it worked perfectly, and all but one would prefer a perfect full body motion system over a hand-held controller.

Mirror's Edge Experts. Those who had experienced Mirror's Edge indicated they didn't feel any more engaged using full body motion than they did using a controller. There were split responses on whether it was more difficult to play the game with the body or the controller. In general, they agreed that the body took less concentration and all agreed it was easier to use the body rather than the controller.

\subsection{Discussion}

Based on the results we found several themes in the data.

Participants start with natural locomotion then transition to compensated locomotion when difficulties present themselves. Nine of the fourteen participants started with natural locomotion then realized the limitations of the constrained physical space. This led to some frustration. For example, to perform their first task of traveling to a lower platform, participants took several steps forward, hopped and found themselves very close to the display. This is the point where they reevaluated their method of locomotion.

Guideline: Design the environment to quickly challenge natural locomotion and then support the switch to compensated locomotion.

Guideline: Expect running in place to be the compensating locomotion technique.

Naturalness was retained for smaller locomotion that fit into the physical space. Although natural locomotion was quickly abandoned for controlling travel, all participants used natural locomotion for short distances of a step or two. Interestingly, participants did not recall this, even during the postexperiment interview. Orientation remained constrained.

Guideline: Retain the ability for natural locomotion in short travel tasks.

Steering also required compensation and two very similar symbolic gestures addressed this. Before developing a compensating steering gesture, participants would find themselves with their back to the display, straining their necks to see. Participants then experimented with several forms of compensating steering, eventually settling on two symbolic gestures: shoulder twist and body rotate and return.

Guideline: Design environments to challenge participant's steering early but unlike locomotion, expect to have to guide them to a particular technique.

Gaze control gestures were controlled by head turns; surprising, as this was different from steering gestures. Of the many participant gaze control gestures, head turning was the most common, and the others accompanied this. We found that gaze control gestures were used when the user wanted to look around in the general direction they were already facing. If the participant wanted to gaze in a direction significantly offset from their current direction, they would then use a steering gesture to change their facing direction to avoid neck and eye strain.

Guideline: Use head orientation to control user's gaze and the body's orientation to control steering.

Desipite pointing's intuitiveness, it was not heavily used, probably due to the arm's use in other gestures. Bowman reports hand-directed steering, or pointing, to be significantly faster than gaze-directed steering in terms of relative motion to an object because changes in direction can be made 
"on the fly" [3]. A relative motion is to spot an object in a virtual world and move to look at it from a specific view point relative to that object. Pointing is powerful because it can either be coupled or independent with gaze as needed. In our results, however, we see little occurrence of pointing and significant use of gaze-directed steering. We believe that pointing wasn't prominent because the arms were frequently busy with other interactions.

Guideline: Full body gestures may have cross interference therefore care should be taken in assigning functions.

Participants differentiated between gestures even when it was not necessary in game play. There are are many interactions mapped to a single button on a controller for most video games. Interestingly, participants create multiple gestures for the same task, specifically for details of the interaction. For example, to distinguish jumping up from jumping down participants raised their hands straight up. Guideline: Full body interfaces may have more gestures, but participants readily understand them and can use them.

Body-centric control of Faith may have helped participants explore gestures. Participants felt that it was quite easy to come up with gestures for these interactions (see Table 1: Q6), and participants who had previously played Mirror's Edge with a hand-held controller thought full body motion was much easier to learn than a controller (see Table 1: Q17). It is likely this occurred because every control situation in the game was derived from a natural motion. This may not have been the case if the participants were required to come up with gestures for unnatural and nonbody-centric video game interactions such as casting spells, using in-game objects, or selecting items in a menu.

New travel gestures can result from the breaking of a natural assumption [15]. So, we simply need to list our assumptions about reality and then break them. Participants, in a reflective manner, might have used this to generate their observed travel gestures. For example, jumping gestures broke the assumption between real and in-game forward motion, climbing gestures break the assumption that the user needs to push off or grip something, sliding gestures break the assumption of a momentum requirement, etc.

Guideline: Predicting possible player control gestures may be achieved by Pierce's assumption breaking methodology.

The fatigue resulting from these gestures will have to be accounted for in gameplay. While participants were neutral regarding the fatigue of the no more than 15 minute session (Q1), typical video gaming sessions can last much longer. Even short but intense physical activity during games can lead to fatigued players [20]. We can imagine scenarios in longer-term play where game controllers are used and only "boss" or other special situations arise where the full body gestures are required. Alternatively, gameplay could shift to shorter-term play or group play, allowing for rest periods. One method might be heavy use of cut scenes and large movie-like sequences in a choose-your-own-adventure style game. Lastly, this fatigue might be beneficial for explicitly creating a physical game.

Guideline: Fatigue needs to be managed as a part of the design of a full body video game.

\begin{tabular}{|l|l|}
\hline \multicolumn{2}{|c|}{ Proposed Gesture Set } \\
\hline Task & Technique \\
\hline Translation (extended) & run in place \\
\hline Translation (local) & unconstrained steps \\
\hline Orientation & body rotate and return \\
\hline Combat & punch and kick \\
\hline Slide & duck \\
\hline Balance & arms out and lean \\
\hline Climb Pole & one arm over the other \\
\hline Climb Up (from hang) & arms push down \\
\hline Climb Up (ladder) & alternating arms \\
\hline Jump (low) & hop, no arms \\
\hline Jump (medium) & hop, arms out front \\
\hline Jump (high) & hop, arms up \\
\hline
\end{tabular}

Table 5: The proposed gesture set takes into account the constraints of the physical performance space.

\subsection{Proposed Gesture Set}

The results show that there is a very intuitive travel control system uncovered by the participants incorporating:

- Constrained Extended Translation

- Unconstrained Local Translation

- Constrained Orientation

- Gaze and Body directed-steering

These gestures are listed in Table 5. Each gesture is egocentric and derived from natural human motion. Not only were these techniques commonly used by our participants, but we feel they are applicable to the beginner, casual, and hardcore game players. Even though few participants attempted kicking, combat includes punching and kicking because both are tasks that can be performed in the game. We did not include a gesture for wall running because little feedback was acquired on this task and during interview, all subjects who performed the side jump technique said it was uncomfortable or difficult to execute.

\section{CONCLUSIONS}

This paper has presented guidelines for designing full body video game intefaces using an exploratory Wizard-of-Oz methodology applied to the action-adventure game Mirror's Edge. The importance between constrained and unconstrained techniques were highlighted. Constrained travel techniques were defined as those performable in a confined space, such as a living room with a game console setup. These techniques are used for extended translation and important aspects include ease of use and concise mappings from user intent to system action. Unconstrained travel techniques were defined as those performed in real-world travel, such as walking. These techniques are effective for only a few steps due to gaming setup constraints. Orientation is also constrained due to the inability to see the television if oriented away from it. Our study, observed an alternative to gaze-directed travel, that of body turn and a back to center gesture. This is important due to the frequent hand use for other interactive tasks. Gaze-directed travel was still observed for granular control of the already displayed viewing area. Lastly, we proposed an initial full body interface specific to the game Mirror's Edge. The interface's gestures are based on human naïve interactions with real environments. Future work remains to see if metaphorical gesture sets would be more effective. 


\section{REFERENCES}

[1] J. N. Bott, J. G. Crowley, and J. J. LaViola, Jr. Exploring 3d gestural interfaces for music creation in video games. In FDG '09: Proceedings of the 4 th International Conference on Foundations of Digital Games, pages 18-25, New York, NY, USA, 2009. ACM.

[2] D. A. Bowman, J. Chen, C. A. Wingrave, J. F. Lucas, A. Ray, N. F. Polys, Q. Li, Y. Haciahmetoglu, J.-S. Kim, S. Kim, R. Boehringer, and T. Ni. New directions in 3d user interfaces. IJVR, 5(2):3-14, 2006.

[3] D. A. Bowman, D. Koller, and L. F. Hodges. Travel in immersive virtual environments: An evaluation of viewpoint motion control techniques. In Proceedings of the Virtual Reality Annual International Symposium, pages 45-52, 1997.

[4] D. A. Bowman, D. Koller, and L. F. Hodges. A methodology for the evaluation of travel techniques for immersive virtual environments. Journal of the Virtual Reality Society, 3:120-131, 1998.

[5] D. A. Bowman, E. Kruijff, J. J. LaViola, and I. Poupyrev. 3D User Interfaces: Theory and Practice. Addison Wesley Longman Publishing Co., Inc., Redwood City, CA, USA, 2004.

[6] E. Burns, A. T. Panter, M. R. McCallus, and F. P. Brooks, Jr. The hand is slower than the eye: A quantitative exploration of visual dominance over proprioception. In $V R$ '05: Proceedings of the 2005 IEEE Conference 2005 on Virtual Reality, pages 3-10, Washington, DC, USA, 2005. IEEE Computer Society.

[7] B. Buxton. Sketching User Experiences: Getting the Design Right and the Right Design. Morgan Kaufmann, first edition, March 2007.

[8] E. Charbonneau, A. Miller, C. Wingrave, and J. J. LaViola, Jr. Understanding visual interfaces for the next generation of dance-based rhythm video games. In Sandbox '09: Proceedings of the 2009 ACM SIGGRAPH Symposium on Video Games, pages 119-126, New York, NY, USA, 2009. ACM.

[9] D. Edwardes. The Parkour and Freerunning Handbook. It Books, New York, NY, USA, 2009.

[10] V. Interrante, B. Ries, and L. Anderson. Seven league boots: A new metaphor for augmented locomotion through moderately large scale immersive virtual environments. 3D User Interfaces, 0:null, 2007.

[11] H. Iwata and T. Fujii. Virtual perambulator: A novel interface device for locomotion in virtual environment. In VRAIS '96: Proceedings of the 1996 Virtual Reality Annual International Symposium (VRAIS 96), page 60, Washington, DC, USA, 1996. IEEE Computer Society.

[12] L. Kratz, M. Smith, and F. J. Lee. Wiizards: 3d gesture recognition for game play input. In Future Play '07: Proceedings of the 2007 conference on Future Play, pages 209-212, New York, NY, USA, 2007. ACM.

[13] P. Maes, T. Darrell, B. Blumberg, and A. Pentland. The alive system: wireless, full-body interaction with autonomous agents. Multimedia Syst., 5(2):105-112, 1997.

[14] J. Payne, P. Keir, J. Elgoyhen, M. McLundie, M. Naef, M. Horner, and P. Anderson. Gameplay issues in the design of spatial $3 \mathrm{~d}$ gestures for video games. In CHI '06: CHI '06 extended abstracts on Human factors in computing systems, pages 1217-1222, New York, NY, USA, 2006. ACM.

[15] J. S. Pierce and R. Pausch. Generating 3d interaction techniques by identifying and breaking assumptions. Virtual Real., 11(1):15-21, 2007.

[16] T. Shiratori and J. K. Hodgins. Accelerometer-based user interfaces for the control of a physically simulated character. In SIGGRAPH Asia '08: ACM SIGGRAPH Asia 2008 papers, pages 1-9, New York, NY, USA, 2008. ACM.

[17] M. Slater, M. Usoh, and A. Steed. Taking steps: the influence of a walking technique on presence in virtual reality. ACM Trans. Comput.-Hum. Interact., 2(3):201-219, 1995.

[18] J. P. Spradley. The Ethnographic Interview. Wadsworth Group, Bellmont, CA, USA, 1979.

[19] A. Wexelblat. An approach to natural gesture in virtual environments. ACM Trans. Comput.-Hum. Interact., 2(3):179-200, 1995.

[20] B. Williamson, C. Wingrave, and J. Laviola. Realnav: Exploring natural user interfaces for locomotion in video games. In To Appear in Proceedings of IEEE Symposium on 3D User Interfaces 2010, 2010.

[21] C. Wingrave, B. Williamson, P. Varcholik, J. Rose, A. Miller, E. Charbonneau, J. Bott, and J. LaViola. Wii remote and beyond: Using spatially convenient devices for 3duis. IEEE Computer Graphics and Applications, 30(2):24-38, 2010.

[22] C. A. Wingrave, R. Tintner, B. N. Walker, D. A. Bowman, and L. F. Hodges. Exploring individual differences in raybased selection; strategies and traits. In VR '05: Proceedings of the 2005 IEEE Conference 2005 on Virtual Reality, pages 163-170, Washington, DC, USA, 2005. IEEE Computer Society.

[23] R. B. Zajonc. Social facilitation. Science, 149:269-274, 1965. 